\title{
The first night effect in multiple sclerosis patients undergoing home-based polysomnography
}

This article was published in the following Dove Press journal:

Nature and Science of Sleep

\section{Christian Veauthier ${ }^{\prime}$ \\ Sophie K Piper ${ }^{2,3}$ \\ Gunnar Gaede ${ }^{4}$ \\ Thomas Penzel 1,5 \\ Friedemann Paul ${ }^{6,7}$ \\ 'Interdisciplinary Center of Sleep Medicine, Charité-Universitätsmedizin Berlin, Corporate Member of Freie Universität Berlin, Humboldt- Universität zu Berlin, and Berlin Institute of Health, Berlin, Germany; ${ }^{2}$ Charité-Universitätsmedizin Berlin, Corporate Member of Freie Universität Berlin, Humboldt-Universität zu Berlin, and Berlin Institute of Health, Institute of Biometry and Clinical Epidemiology, Berlin, Germany; ${ }^{3}$ Berlin Institute of Health (BIH), Berlin, Germany; ${ }^{4}$ Department of Neurology, St. Joseph Krankenhaus Berlin-Weissensee, Berlin, Germany; International Clinical Research Center, St. Anne's University Hospital Brno, Brno, Czech Republic; ${ }^{6}$ NeuroCure Clinical Research Center, Charité-Universitätsmedizin Berlin, Corporate Member of Freie Universität Berlin, Humboldt-Universität zu Berlin, and Berlin Institute of Health, Berlin, Germany; ${ }^{7}$ Experimental and Clinical Research Center, Max Delbrueck Center for Molecular Medicine and Charité-Universitätsmedizin Berlin, Corporate Member of Freie Universität Berlin, Humboldt-Universität zu Berlin, and Berlin Institute of Health, Berlin, Germany}

Correspondence: Christian Veauthier Charité-Universitätsmedizin Berlin, Interdisciplinary Center of Sleep Medicine, Luisenstraße 13, I0II7 Berlin, Germany

Tel +49304505 I 3120

$\mathrm{Fax}+4932122828668$

Email christian.veauthier@charite.de
Background: The first night effect (FNE) is a polysomnography (PSG) habituation effect in the first of several consecutive in-laboratory PSGs (I-PSGs). The FNE is caused by the discomfort provoked by electrodes and cables and the exposure to an unfamiliar environment. A reverse FNE (RFNE) with an improved sleep in the first night is characteristic of insomnia, presumably because the video PSG in the sleep laboratory leads to a decrease in the negatively toned cognitive activity. Therefore, two or more I-PSGs are required for an accurate diagnosis. Although the FNE is well documented in I-PSG, little is known about the FNE and the RFNE in home-based PSGs (H-PSGs).

Methods: This is a retrospective analysis of a recently published cross-sectional study using H-PSG. Sixty-three consecutive patients suffering from multiple sclerosis (MS) were investigated by two consecutive H-PSGs without video. The differences between the first and second H-PSGs were analyzed. The patients were classified into four subgroups: no sleep disorder, insomnia, sleep-related breathing disorders (SRBDs), and periodic limb movement disorder or restless legs syndrome (PLMD/RLS).

Results: MS patients suffering from insomnia showed no RFNE. MS patients with SRBD or PLMD/RLS showed no reduced sleep efficiency but significantly less slow wave sleep. Furthermore, SRBD patients showed significantly less non-rapid eye movement (NREM) sleep, and PLMD/RLS patients were significantly awake longer in the first night after sleep onset (increased wake-after-sleep-onset time) and showed a higher rapid eye movement (REM) latency. Conclusion: SRBD and PLMD/RLS patients showed a significant FNE. Two consecutive H-PSGs are required in these patients to obtain a precise hypnogram even in the ambulatory field. In MS patients suffering from insomnia, no RFNE was found, and in insomnia patients one H-PSG seems to be sufficient.

Keywords: insomnia, multiple sclerosis, outpatient sleep studies, polysomnography, sleep latency, wake after sleep onset

\section{Introduction}

Polysomnography (PSG) is the gold standard for diagnosing many different sleep disorders, in particular, obstructive sleep apnea syndrome (OSAS) and is restricted for the diagnosis of only the most severe sleep disorders due to its low availability and high cost. ${ }^{1,2}$ Polygraphy (PG) is often used as an alternative diagnostic technique for diagnosing OSAS, ${ }^{3}$ but it is insufficient to rule out OSAS. ${ }^{4}$ Actigraphy is often used to estimate sleep-wake time and to measure body position. ${ }^{5-7}$ To reduce the numbers of PSG and save resources, new technologies such as automated electroencephalographybased single-channel sleep-wake detection algorithms or actigraphy combined with 
respiratory effort have been described, which are not yet part of routine clinical practice, and up to date PSG remains the gold standard. , $^{8}$

In one recently published study, 187 adults suffering from sleepiness or tiredness who had undergone previously ambulant PG and were considered to be normal were afterward investigated by PSG: the authors found at least mild OSAS in $90 \%$, and 64\% showed even moderate-to-severe OSAS. ${ }^{4}$ Even if the authors concluded that PG is insufficient to rule out OSAS when the respiratory events are mainly associated with arousals, due to the increasing demand for in-laboratory PSG (I-PSG) and long waiting lists of the most sleep centers, PG is nevertheless increasingly utilized in this issue. ${ }^{10}$ The advantage of self-administrated home-based PSG (H-PSG) recordings is that they are less time-consuming and less cost-intensive. Bruyneel and Ninane ${ }^{11}$ reviewed recently six prospective randomized crossover studies comparing $\mathrm{H}-\mathrm{PSG}$ to I-PSG. These six studies showed that unattended H-PSG shows a low failure rate despite the absence of supervision, and it is sufficiently accurate for OSAS diagnosis. Moreover, the quality of patients' sleep tends to be better at home. ${ }^{11}$ PSGs were usually performed in the sleep laboratory (I-PSG). Several I-PSG studies in healthy volunteers (HVs) and insomniacs showed, on the first PSG recording, a lower total sleep time (TST) and reduced rapid eye movement (REM) sleep with increased sleep onset latencies (SOLs) and REM sleep latencies (RSLs), lower sleep efficacy (SE), and higher wake-time-after-sleep-onset (WASO) time as compared to the second PSG, whereas the total amount of non-REM (NREM) sleep remains unchanged. ${ }^{12,13}$ This so-called first night effect (FNE) is caused by the discomfort provoked by electrodes and cables and the exposure to an unfamiliar environment. ${ }^{12,14}$ It is not known whether and to what extent there is an FNE in H-PSG.

It has generally been considered that H-PSG in contrast to I-PSG does not provoke an FNE because the change in environment has a larger impact than the H-PSG itself. ${ }^{14}$ Repeated studies on overall 100 persons failed to demonstrate an FNE in HVs and insomniacs at home. ${ }^{15-17}$ However, two studies showed FNE in the home environment as well, comparable to recordings in the sleep laboratory - although to a lesser extent: Le Bon et $\mathrm{al}^{14}$ investigated $26 \mathrm{HVs}$ at home, and they found a classic FNE between night 1 and night 2 regarding SE, WASO time, awakenings (AWAs), and REM sleep, whereas the duration of NREM sleep remained the same. Wauquier et $\mathrm{al}^{18}$ found an FNE in two consecutive 24-hour ambulatory PSG home sleep-wake recordings of 10 elderly persons with a mean age of 86 years.
Moreover, Blackwell et a ${ }^{19}$ used actigraphy to quantify the sleep disruption level caused by H-PSG. They investigated 778 male subjects aged 65 years and older from a population-based study by H-PSG, and the simultaneous actigraphy was performed on the PSG night and three subsequent nights. ${ }^{19}$ Sleep on the first H-PSG night was significantly worse than in the following nights (less actigraphically measured TST and SE, more WASO and SOL). Among others, older age was associated with greater sleep disruption on the PSG night.

In 2011, we published the results of the first PSG crosssectional trial in multiple sclerosis (MS) patients, which we performed over two consecutive nights at home. ${ }^{20}$ The aim of the present retrospective data analysis was to evaluate a possible FNE in our original cohort. Moreover, we analyzed the so-called "reversed" or "paradoxical" FNE (RFNE). ${ }^{21}$ The RFNE is characterized in insomniacs by the observation of decreased SOL, decreased REM latency, a higher percentage of REM, and greater SE in the first night compared to the second night.

\section{Patients and methods Participants and procedures}

In this study, we investigated 66 consecutive MS patients by two H-PSGs using the Rechtschaffen and Kales (R\&K) criteria for classification of sleep stages, the Coleman criteria for classifying periodic leg movement (PLM), and the diagnostic guidelines of the Task Force of the American Academy of Sleep Medicine (AASM) for respiratory events. ${ }^{20,22-25}$ In the present analysis, 63 patients were included (due to missing data in three patients of the first night). We retrospectively analyzed the PSG data from the two consecutive nights (PLM index [PLMI] per hour sleep, PLM arousal index [PLM-AI] per hour sleep), SE (percentage of TST per time spent in bed [TiB]), percentage of slow wave sleep (NREM 3 and 4) per TiB, percentage of NREM 1 and 2 sleep per TiB, percentage of REM sleep per TiB, percentage of NREM sleep per TiB, SOL in minutes, WASO in minutes, number of AWAs, and arousal indexes (AIs). This study was approved by the local ethics committees (institutional review board of the University of Greifswald, Germany; vote number BB 03/08), and all the participants gave written informed consent prior to the assessment.

\section{Statistical analyses}

The results are reported as frequencies and percentages for categorical variables and mean and standard deviation (SD) for sufficiently normally distributed continuous data 
(|skewness $\mid<1)$ or median and limits of the inter-quartile range (IQR) for quantitatively skewed variables. Changes $(\Delta)$ in sleep parameters were calculated in different subgroups as the difference between the second and first night and compared using Wilcoxon signed-rank test (Tables 1 and 2) within each sleep disorder subgroup. Differences in sleep parameter changes between the four subgroups were tested using Kruskal-Wallis test. All tests were two sided with a significant level of 0.05 . To detect associations between changes in sleep parameters and patients' age, we used Spearman's rank correlations for univariate analyses within each subgroup. A correlation coefficient close to zero means that the change between the two nights does not or only very weakly correlate with age. The larger the correlation coefficient (maximum possible absolute value is $|1|$ ), the greater the impact of age on the changes in H-PSG parameters. Scatter plots are given to illustrate correlations. We rated Spearman's rank correlations (Spearman's rho) as follows: for values equal or greater than 0.4 , the correlation is at least moderate or stronger; for values lower than 0.4 , the correlation is weak and considered not of relevance. No further adjustment for multiple comparisons has been applied. All tests should be understood as constituting exploratory data analysis.

\section{Results}

Baseline characteristics and demographic data are given in Table 1. Due to the RFNE in insomniacs, the FNE was not calculated in the entire cohort but calculated separately in the four sleep disorder subgroups. The results are summarized in Table 2. Apart from an increased number of AWAs in the first night, MS patients without sleep disorders showed no significant differences between the two nights. MS patients suffering from sleep-related breathing disorders (SRBDs, six OSAS patients and one patient suffering from central sleep apnea) showed significantly less NREM sleep and slow wave sleep in the first night $(12.5 \%$ and $2.8 \%$, respectively) compared to the second night (55.2\% and $8.3 \%$, respectively; $P=0.043$ and $P=0.028$, respectively). Moreover, SRBD patients showed 30 minutes more WASO in the first night compared to the second night $(P=0.176)$. Periodic limb movement disorder or restless legs syndrome (PLMD/RLS) patients showed less slow wave sleep in the first night ( $7.2 \%$ vs $9.3 \% ; P=0.008$ ) and, furthermore, significantly more WASO ( 80 vs 69 minutes; $P=0.029$ ) and a higher REM latency (79 vs 61 minutes; $P=0.027$ ) in the first night compared to the second night. Insomniacs showed changes between the two consecutive nights, consistent with the described RFNE in the literature although they failed to reach statistical significance in our data set: shorter SOL (41 vs 54 minutes; $P=0.705)$ in the first night and a higher SE (72.7\% vs $70.2 \% ; P=0.407)$.

Table 3 summarizes the Spearman's rank correlations between changes in sleep parameters between night 1 and night $2($ Delta $=\Delta)$ and age. Only a few moderate correlations were found. In MS patients without sleep disorders, the change in AI was significantly correlated with age

Table I Baseline characteristics

\begin{tabular}{|c|c|c|c|c|c|c|c|}
\hline & & & $\begin{array}{l}\text { Insomnia } \\
(n=17)\end{array}$ & $\begin{array}{l}\text { No sleep disorder } \\
(n=17)\end{array}$ & $\begin{array}{l}\text { SRBD } \\
(n=7)\end{array}$ & $\begin{array}{l}\text { PLMD/RLS } \\
(n=22)\end{array}$ & $\begin{array}{l}\text { Total } \\
(\mathrm{N}=63)\end{array}$ \\
\hline Sex & \multicolumn{2}{|l|}{ Female, N (\%) } & $13(76.5)$ & $14(82.4)$ & $5(7 I .4)$ & II (50.0) & $43(68.3)$ \\
\hline Age (years) & \multicolumn{2}{|l|}{ Mean (SD) } & $46(10)$ & $36(9)$ & $51(10)$ & $44(9)$ & $43(10)$ \\
\hline Time since onset (years) & \multicolumn{2}{|l|}{ Median (IQR) } & $9(7-22)$ & $5(4-11)$ & $10(3-24)$ & II (4-18) & $9(4-18)$ \\
\hline Expanded Disability Status Scale & \multicolumn{2}{|l|}{ Median (IQR) } & $2(2-3)$ & $2(2)$ & $4(3-6)$ & $2(2-4)$ & $2(2-4)$ \\
\hline Beck Depression Inventory & \multicolumn{2}{|l|}{ Median (IQR) } & $15(12-23)$ & $5(2-8)$ & $14(\mathrm{II}-33)$ & $9(6-16)$ & $10(6-18)$ \\
\hline Modified Fatigue Impact Scale & \multicolumn{2}{|l|}{ Median (IQR) } & $48(44-54)$ & $20(2-3 I)$ & $57(45-64)$ & $33(19-48)$ & $38(20-52)$ \\
\hline Fatigue Severity Scale & \multicolumn{2}{|l|}{ Median (IQR) } & $5.7(5.1-6.5)$ & $3.1(1.4-4.4)$ & $6.3(5.7-6.9)$ & $4.0(2.9-5.4)$ & $4.7(3.1-6.3)$ \\
\hline \multirow[t]{8}{*}{ Disease-modifying therapy } & Missings & $\mathrm{n}$ & 1 & 1 & 0 & 4 & 6 \\
\hline & Copaxone & $\mathrm{n}(\%)$ & $3(18.8)$ & $9(56.3)$ & $3(42.9)$ & $5(27.8)$ & $20(35.1)$ \\
\hline & Cortisone plus & $\mathrm{n}(\%)$ & 0 & $\mathrm{I}(6.3)$ & 0 & 0 & $\mathrm{I}(\mathrm{I} .8)$ \\
\hline & Interferon & $\mathrm{n}(\%)$ & $7(43.8)$ & $5(3 I .3)$ & $3(42.9)$ & $7(38.9)$ & $22(38.6)$ \\
\hline & IVIG & $\mathrm{n}(\%)$ & 0 & $I(6.3)$ & 0 & $I(5.6)$ & $2(3.5)$ \\
\hline & No therapy & $\mathrm{n}(\%)$ & $3(18.8)$ & 0 & I (I4.3) & $5(27.8)$ & $9(15.8)$ \\
\hline & Mitoxantrone & $\mathrm{n}(\%)$ & $2(12.5)$ & 0 & 0 & 0 & $2(3.5)$ \\
\hline & Natalizumab & $\mathrm{n}(\%)$ & $\mathrm{I}(6.3)$ & 0 & 0 & 0 & 1 \\
\hline Antidepressants & Yes & $\mathrm{n}(\%)$ & $5(29.4)$ & $5(29.4)$ & 0 & $4(18.2)$ & $14(22.2)$ \\
\hline Psychopharmaka & Yes & $\mathrm{n}(\%)$ & $5(29.4)$ & $I(5.9)$ & $2(28.6)$ & $8(36.4)$ & $16(25.4)$ \\
\hline
\end{tabular}

Abbreviations: IVIG, polyvalent intravenous globulin; PLMD, periodic limb movement disorder; RLS, restless legs syndrome; SRBD, sleep-related breathing disorder. 
Table 2 Hypnogram parameters: night I and median change to night 2 ( $\Delta$ : night 2-night I)

\begin{tabular}{|c|c|c|c|c|}
\hline & Insomnia $(n=\mid 7)$ & $\begin{array}{l}\text { No sleep } \\
\text { disorder }(n=17)\end{array}$ & SRBD $(n=7)$ & $\begin{array}{l}\text { PLMD/RLS } \\
(n=22)\end{array}$ \\
\hline $\begin{array}{l}\text { Al per hour sleep (first night) } \\
\Delta \text { second night vs first night }\end{array}$ & $\begin{array}{l}21.0(14.7-29.3) \\
-1(-6 \text { to } 3) \\
P=0.737\end{array}$ & $\begin{array}{l}I 8.9(|3 .|-27.0) \\
-I(-3 \text { to } 2) \\
P=0.959\end{array}$ & $\begin{array}{l}23.6(I I .0-40.6) \\
-3(-3 \text { to } 6) \\
P=0.735\end{array}$ & $\begin{array}{l}15.9(12.3-46.0) \\
0(-3 \text { to } I) \\
P=0.052\end{array}$ \\
\hline $\begin{array}{l}\text { WASO in minutes (first night) } \\
\Delta \text { second night vs first night }\end{array}$ & $\begin{array}{l}83(57-136) \\
-7(-2 \mid \text { to } 36) \\
P=0.962\end{array}$ & $\begin{array}{l}68(53-85) \\
-12(-30 \text { to } 0) \\
P=0.112\end{array}$ & $\begin{array}{l}174(74-2 \mid 8) \\
-30(-85 \text { to } 3) \\
P=0.176\end{array}$ & $\begin{array}{l}80(55-109) \\
-I I(-3 I \text { to } 0) \\
P=0.029\end{array}$ \\
\hline $\begin{array}{l}\text { Number of sleep stage changes (first night) } \\
\Delta \text { second night vs first night }\end{array}$ & $\begin{array}{l}I 57(|||6-| 88) \\
-2 \mid(-26 \text { to } 26) \\
P=0.636\end{array}$ & $\begin{array}{l}163(138-206) \\
-7(-51 \text { to } 2) \\
P=0.115\end{array}$ & $\begin{array}{l}153(109-218) \\
10(-24 \text { to } 22) \\
P=0.799\end{array}$ & $\begin{array}{l}146(|23-| 78) \\
2(-36 \text { to } 24) \\
P=0.970\end{array}$ \\
\hline $\begin{array}{l}\text { Numbers of AWAs (first night) } \\
\Delta \text { second night vs first night }\end{array}$ & $\begin{array}{l}25(22-35) \\
0(-5-7) \\
P=0.532\end{array}$ & $\begin{array}{l}27(17-39) \\
-5(-9 \text { to } 2) \\
P=0.04 \mid\end{array}$ & $\begin{array}{l}34(27-48) \\
2(0 \text { to } 7) \\
P=0.498\end{array}$ & $\begin{array}{l}27(17-32) \\
0(-9 \text { to } 5) \\
P=0.808\end{array}$ \\
\hline $\begin{array}{l}\text { SE in percent of TSiB (first night) } \\
\Delta \text { second night vs first night }\end{array}$ & $\begin{array}{l}72.7(65.2-8 \mid .4) \\
-2.5(-10.2 \text { to } 6.2) \\
P=0.407\end{array}$ & $\begin{array}{l}82.9(78.2-86.9) \\
I .8(-1.8 \text { to } 4.2) \\
P=0.3 \mid 3\end{array}$ & $\begin{array}{l}66.4(59.4-84.4) \\
4.2(-7.8 \text { to } 19.0) \\
P=0.310\end{array}$ & $\begin{array}{l}76.5(69.9-83.4) \\
-0.1(-3.5 \text { to } 5.8) \\
P=0.986\end{array}$ \\
\hline $\begin{array}{l}\mathrm{NI} \text { and N2 in percent of TB (first night) } \\
\Delta \text { second night vs first night }\end{array}$ & $\begin{array}{l}50.8(43.8-60.9) \\
-3.6(-12.8 \text { to } 3.6) \\
P=0.256\end{array}$ & $\begin{array}{l}52.3(46.7-62.1) \\
0(-7.0 \text { to } 7.8) \\
P=0.959\end{array}$ & $\begin{array}{l}47.4(36.2-5 \mid .7) \\
\text { II.0 (-6.2 to I2.4) } \\
P=0.398\end{array}$ & $\begin{array}{l}52.5(47 . I-64 . I) \\
0(-6.5 \text { to } 5.1) \\
P=0.502\end{array}$ \\
\hline $\begin{array}{l}\text { REM sleep in percent of TB (first night) } \\
\Delta \text { second night vs first night }\end{array}$ & $\begin{array}{l}12.2(8.2-16.0) \\
0.7(-1.9 \text { to } 4.7) \\
P=0.670\end{array}$ & $\begin{array}{l}\mid 3.5(\mid 0.0-18.0) \\
0.5(-3.6 \text { to } 6.4) \\
P=0.443\end{array}$ & $\begin{array}{l}\text { I3.I (6.8-I8.3) } \\
-0.1(-4.3 \text { to } 6.2) \\
P=0.735\end{array}$ & $\begin{array}{l}\mid 4.3(7.7-\mid 5.8) \\
0.8(-2.7 \text { to } 5.3) \\
P=0.390\end{array}$ \\
\hline $\begin{array}{l}\text { SWS in percent of TB (first night) } \\
\Delta \text { second night vs first night }\end{array}$ & $\begin{array}{l}7.4(3.7-12.0) \\
2.2(-1.1 \text { to } 7.0) \\
P=0.079\end{array}$ & $\begin{array}{l}\text { II.4 (7.6-16.5) } \\
4.0(-0.6 \text { to } 6.4) \\
P=0.070\end{array}$ & $\begin{array}{l}8.3(5.8-10.7) \\
2.8(1.4-\mid 4.1) \\
P=0.028\end{array}$ & $\begin{array}{l}7.2(3.8-12.0) \\
2.1(0.5-6.7) \\
P=0.008\end{array}$ \\
\hline $\begin{array}{l}\text { NREM sleep in percent of TB (first night) } \\
\Delta \text { second night vs first night }\end{array}$ & $\begin{array}{l}61.6(53.0-70.3) \\
1.3(-5.4 \text { to } 10.3) \\
P=0.943\end{array}$ & $\begin{array}{l}65.9(61.1-69.4) \\
4.0(-3.6 \text { to } 6.9) \\
P=0.177\end{array}$ & $\begin{array}{l}55.2(45.9-65.0) \\
\mid 2.5(4.6 \text { to } \mid 4.1) \\
P=0.043\end{array}$ & $\begin{array}{l}62.7(58.6-67.2) \\
5.2(-5.1 \text { to } 7.9) \\
P=0.263\end{array}$ \\
\hline $\begin{array}{l}\text { REM latency in minutes (first night) } \\
\Delta \text { second night vs first night }\end{array}$ & $\begin{array}{l}75(58-104) \\
-5(-28 \text { to } 50) \\
P=0.649\end{array}$ & $\begin{array}{l}86(49-140) \\
-10(-63 \text { to } 18) \\
P=0.408\end{array}$ & $\begin{array}{l}83(38-124) \\
-7(-53 \text { to } 45) \\
P=0.866\end{array}$ & $\begin{array}{l}79(55-116) \\
-18(-38 \text { to } 6) \\
P=0.027\end{array}$ \\
\hline $\begin{array}{l}\text { SOL in minutes (first night) } \\
\Delta \text { second night vs first night }\end{array}$ & $\begin{array}{l}4 \mid(|9-5|) \\
13(-15 \text { to } 16) \\
P=0.705\end{array}$ & $\begin{array}{l}I 7(6-25) \\
-I(-14 \text { to I0) } \\
P=0.938\end{array}$ & $\begin{array}{l}\text { II }(I-2 I) \\
5(-I \text { to I9) } \\
P=0.176\end{array}$ & $\begin{array}{l}27(15-52) \\
0(-15 \text { to } 18) \\
P=0.794\end{array}$ \\
\hline
\end{tabular}

Notes: Significant differences between the second and the first night are marked in bold $(P<0.05$ Wilcoxon signed-rank test). The results are presented as median results. The numbers in parentheses show the IQR.

Abbreviations: Al, arousal index; AWA, awakening; NI/N2, NREM sleep stage I/2; NREM, non-REM; PLMD, periodic limb movement disorder; REM, rapid eye movement; RLS, restless legs syndrome; SOL, sleep onset latency; SRBD, sleep-related breathing disorder; SWS, slow wave sleep; TB, time spent in bed; WASO, wake after sleep onset.

$($ rho $=0.517)$ : older patients showed a larger decrease in AI between the first and second night than younger MS patients without sleep disorders (as a sign of a slight FNE in older patients). In addition, in MS patients suffering from insomnia, the change in REM latency was significantly correlated with age (rho $=-0.620)$ : older patients showed a smaller increase up to a decrease in REM latency. In PLMD/RLS patients, the changes in SOL and REM sleep were significantly correlated with age too (rho $=-0.424$ and rho $=0.451$, respectively). In MS patients suffering from SRBD, there were moderate correlations with age and changes in the number of AWAs $($ rho $=-0.477)$, NREM sleep $($ rho $=0.468)$, NREM sleep $1+2$ (rho $=0.577)$, and SOL (rho $=-0.450)$. Figure 1 illustrates the correlations between age and PSG parameters in different subgroups by scatter plots.

\section{Discussion}

Although the observed FNE in H-PSG was smaller as described using I-PSG and has not been accompanied by a reduced $\mathrm{SE}$, even in an outpatient setting a significant FNE in H-PSG exists. In particular, MS patients suffering from SRBD and PLMD/RLS showed a clear FNE - probably caused by the discomfort provoked by electrodes and cables. In contrast, in MS patients suffering from insomnia, no significant RFNE could be found, similar to other studies which did not show an RFNE in insomniacs in an 
Table 3 Spearman's rank correlations of the FNE with age within each subgroup

\begin{tabular}{|c|c|c|c|c|c|}
\hline \multicolumn{2}{|c|}{ Spearman's rank correlation with age (years) } & \multirow{2}{*}{$\begin{array}{l}\begin{array}{l}\text { Insomnia } \\
(n=17)\end{array} \\
-0.401\end{array}$} & \multirow{2}{*}{$\begin{array}{l}\text { No sleep } \\
\text { disorder }(n=17) \\
-0.517\end{array}$} & \multirow{2}{*}{ 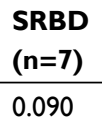 } & \multirow{2}{*}{$\begin{array}{l}\text { PLMD/RLS } \\
(\mathbf{n}=\mathbf{2 2}) \\
-0.276 \\
\end{array}$} \\
\hline$\Delta \mathrm{Al}$ (per hour of sleep) & Correlation coefficient & & & & \\
\hline & $P$-value & 0.111 & 0.034 & 0.848 & 0.214 \\
\hline \multirow[t]{2}{*}{$\triangle$ WASO (minutes) } & Correlation coefficient & 0.418 & -0.004 & 0.108 & 0.156 \\
\hline & $P$-value & 0.095 & 0.987 & 0.818 & 0.488 \\
\hline \multirow{2}{*}{$\begin{array}{l}\Delta \text { Number of sleep stage } \\
\text { changes }\end{array}$} & Correlation coefficient & 0.169 & -0.102 & -0.324 & -0.117 \\
\hline & $P$-value & 0.518 & 0.696 & 0.478 & 0.605 \\
\hline \multirow[t]{2}{*}{$\Delta$ Number of AWAs } & Correlation coefficient & 0.085 & -0.023 & -0.477 & 0.091 \\
\hline & $P$-value & 0.746 & 0.929 & 0.279 & 0.687 \\
\hline \multirow[t]{2}{*}{$\Delta \mathrm{SE}(\%$ of $\mathrm{TSiB})$} & Correlation coefficient & -0.370 & -0.237 & -0.180 & 0.046 \\
\hline & $P$-value & 0.144 & 0.360 & 0.699 & 0.840 \\
\hline \multirow{2}{*}{$\begin{array}{l}\Delta \text { NREM sleep I+2 (\% of } \\
\text { TSiB) }\end{array}$} & Correlation coefficient & -0.448 & 0.121 & 0.577 & -0.302 \\
\hline & $P$-value & 0.072 & 0.644 & 0.175 & 0.171 \\
\hline \multirow{2}{*}{$\begin{array}{l}\Delta \text { Slow wave sleep (N3) } \\
\text { (\% of TSiB) }\end{array}$} & Correlation coefficient & 0.049 & -0.228 & -0.144 & 0.053 \\
\hline & $P$-value & 0.851 & 0.379 & 0.758 & 0.813 \\
\hline \multirow{2}{*}{$\begin{array}{l}\Delta \text { NREM sleep }(\% \text { of } \\
\text { TSiB) }\end{array}$} & Correlation coefficient & -0.080 & 0.002 & 0.468 & -0.191 \\
\hline & $P$-value & 0.760 & 0.993 & 0.289 & 0.394 \\
\hline \multirow[t]{2}{*}{$\Delta$ REM latency (minutes) } & Correlation coefficient & -0.620 & -0.116 & -0.360 & 0.088 \\
\hline & $P$-value & 0.014 & 0.670 & 0.427 & 0.697 \\
\hline \multirow[t]{2}{*}{$\Delta \mathrm{SOL}$ (minutes) } & Correlation coefficient & 0.286 & 0.274 & -0.450 & -0.424 \\
\hline & $P$-value & 0.266 & 0.286 & 0.310 & 0.049 \\
\hline \multirow[t]{2}{*}{$\Delta$ REM sleep (\% of TSiB) } & Correlation coefficient & -0.068 & -0.324 & -0.144 & $0.45 I$ \\
\hline & $P$-value & 0.796 & 0.205 & 0.758 & 0.035 \\
\hline
\end{tabular}

Notes: Changes in sleep parameters between night I and night 2 were analyzed. Significant differences between the second and the first night are marked in bold $(P<0.05$ Wilcoxon signed-rank test).

Abbreviations: Al, arousal index; AWA, awakening; FNE, first night effect; N3, NREM sleep stage 3; NREM, non-REM; PLMD, periodic limb movement disorder; REM, rapid eye movement; RLS, restless legs syndrome; SE, sleep efficacy; SOL, sleep onset latency; SRBD, sleep-related breathing disorder; TSiB, time spent in bed; WASO, wake after sleep onset.

outpatient setting. ${ }^{12-14}$ The RFNE is usually caused by the unusual environment and the feeling to be under observation. In this study, the patients were not filmed; moreover, they slept at home. This could be the reason why especially in insomniacs no significant RFNE was found.

Newell et $\mathrm{al}^{26}$ compared two consecutive I-PSGs, and the patients were divided into four subgroups: insomnia, SRBD, PLMD/parasomnia, and healthy controls (HCs). When comparing both nights of all four groups separately, the changes between the two I-PSGs were most pronounced in the insomnia group. In particular, in this study, SRBD patients showed in the first night no reduced WASO time, no reduced REM, and no increased sleep latencies (RSL or SOL) - similar to the SRBD patients in our study. Against this background, we can summarize that the FNE in SRBD patients found in our study seems to be very similar to the FNE in the sleep laboratory observed by Newell et al and that this FNE is most likely caused by the cables itself and not by the unfamiliar environment.

On the other hand, MS patients suffering from insomnia showed, in the familiar environment (in contrast to the study by Newell et al), no significant changes between the two consecutive nights. It could therefore be concluded that the RFNE in insomnia patients found in previous studies (using I-PSG in the sleep laboratory) has been probably caused by the unfamiliar environment. This is clinically important: the point in question is, how many H-PSGs should be performed at home in insomnia and SRBD and RLS/PLMD patients. The fact that insomnia patients showed no RFNE in our study argues for only one H-PSG in these patients. Due to the fact that a typical FNE was found in SRBD patients at home, two H-PSGs should be performed for a correct diagnosis. On the other hand, it must be respected that in SRBD patients PSGs (H-PSG or I-PSG) are usually performed for a correct diagnosis of SRBD, and it can be expected that in the clinical routine two H-PSGs will not be routinely required for a correct SRBD diagnosis.

Moreover, in MS patients without sleep disorders, only an increased number of AWAs were found in the first night, what goes along with studies which failed to demonstrate a significant FNE in an outpatient setting in HCs. In MS patients suffering from PLMD/RLS, NREM sleep was reduced in the first night and sleep latencies and WASO time were increased. Therefore, even if the SE and TST showed no significant changes between the two nights, we found smaller changes which corresponded - albeit to a lesser extent - to those observed in I-PSG. 
A

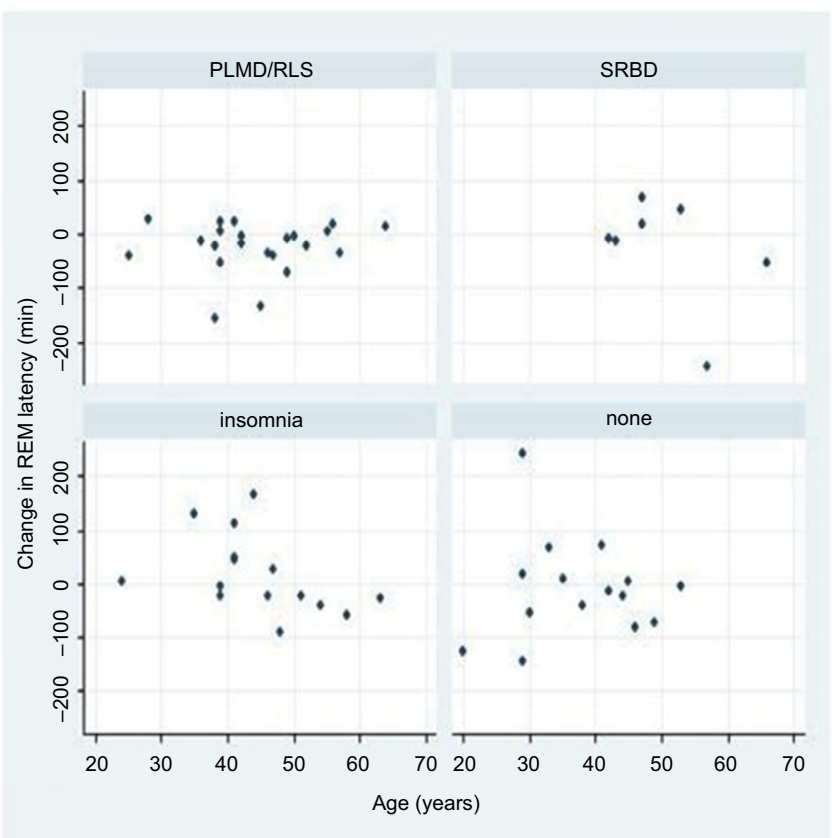

B

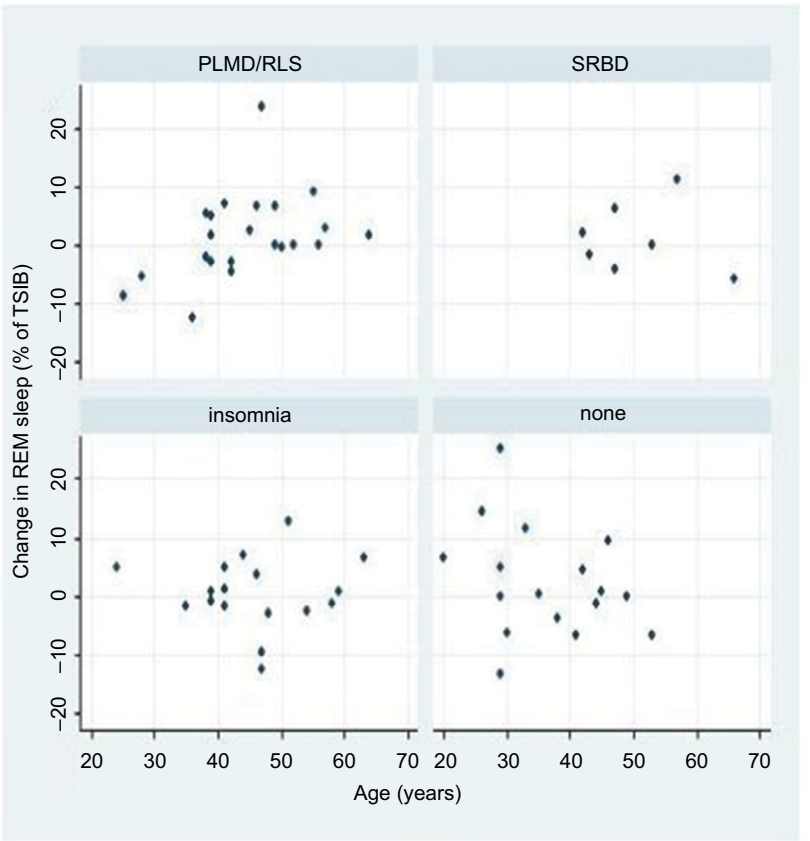

C

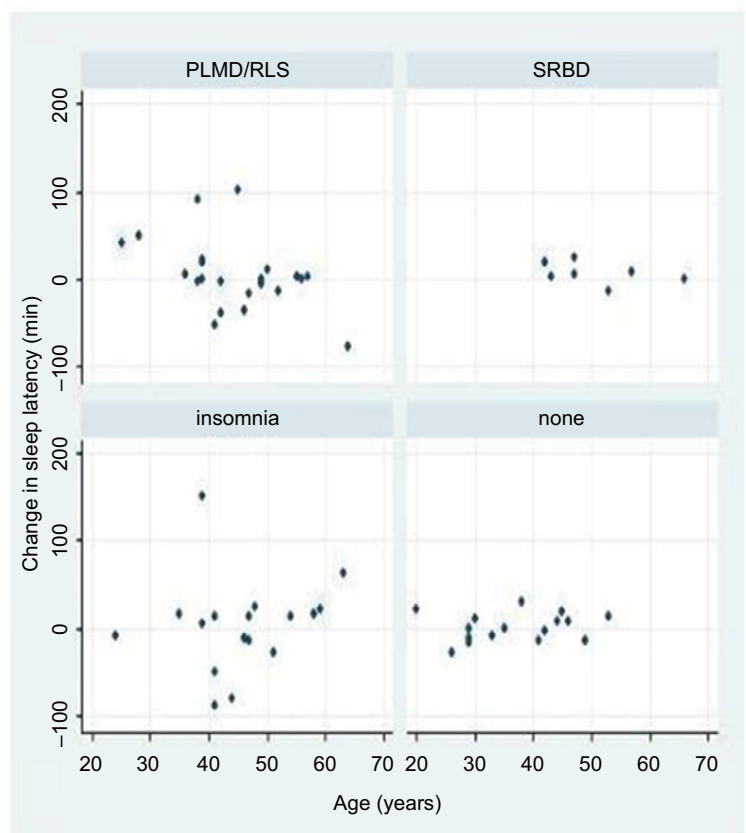

D

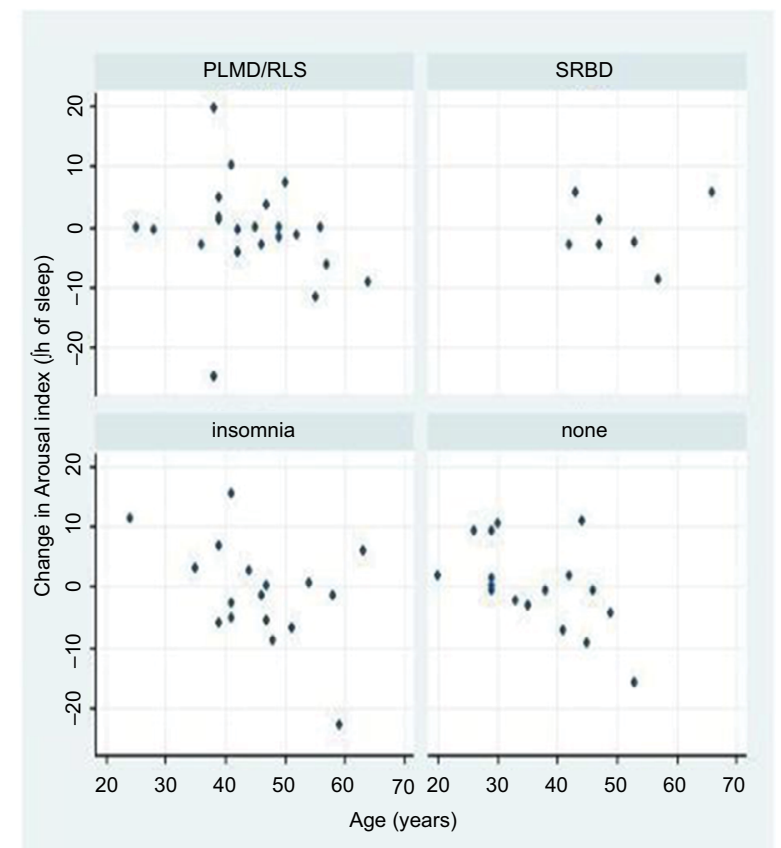

Figure I In some sleep parameters, age-related changes between the two nights were found.

Notes: In MS patients suffering from insomnia, younger age was correlated with an increased REM latency in the first night (A). In MS patients suffering from PLMD or RLS, older patients spent less time in REM sleep in the first night (B), whereas younger patients needed more time to fall asleep in the first night (C). Older MS patients without any sleep disorder showed a larger decrease in arousal between the first and second night, indicating possible indication for a slight FNE in older patients (D). In summary, the age-related changes were relatively small, and older and younger patients were similarly affected although in various parameters: Amongst MS patients suffering from insomnia or PLMD/RLS, the younger the patient the smaller the REM latency and sleep onset latency. In MS patients without sleep disorders, the older the patients the higher the number of arousal in the first night. But, in general, it cannot be said that older or younger patients showed a more pronounced FNE.

Abbreviations: Al, arousal index; FNE, first night effect; MS, multiple sclerosis; PLMD, periodic limb movement disorder; REM, rapid eye movement; RLS, restless legs syndrome; SOL, sleep onset latency; SRBD, sleep-related breathing disorder; TSiB, time spent in bed. 
In summary, MS patients suffering from sleep disturbances who were suffering from SRBD and PLMD/RLS showed a clear FNE in the H-PSG, which was in PLMD/ RLS patients less pronounced than in the sleep laboratory but should be taken into account in all studies investigating sleep in these patients. Due to the fact that only MS patients were investigated in this study, these findings cannot be generalized to the general population. However, a recently published study shows that insomnia comorbid to MS is associated with the same classical psychological factors as insomnia in the general population. ${ }^{27}$ That means that insomnia in MS patients is not different from insomnia in the general population. In our study, MS patients suffering from insomnia showed in the first night a nonsignificantly increased sleep efficiency and a nonsignificantly decreased sleep latency. These findings, even if they are not significant and less pronounced than in the sleep laboratory, correspond to what is described about RFNE in insomnia in the sleep laboratory (it points in the same direction). Therefore, there are no arguments that our findings are specific for the MS population. The small nonsignificant improvement in sleep in MS patients suffering from insomnia in the first night and the observed significant FNE in MS patients suffering from SRBD and RLS/PLMD are in line with the RFNE/FNE in the sleep laboratory described in the literature, and it cannot be expected that these results are caused by the MS itself even if the MS can be theoretically a substantial confounder. Taken together, to investigate the FNE and RFNE in an outpatient setting, larger prospective studies using the new AASM criteria in patients suffering only from sleep disorders without MS and other comorbid diseases should be performed.

\section{Methodical limitations}

This is a retrospective analysis of a cross-sectional study published in Multiple Sclerosis Journal in 2011. The original study began in 2007, in the year in which the new scoring criteria by the AASM were published. The used PSG systems (two of them provided by Weinmann Medical Technology) measured sleep by $\mathrm{C} 3-\mathrm{A} 2$ and $\mathrm{C} 4-\mathrm{C} 1$ electrodes and did not have frontal and occipital electrodes. Therefore, the PSG had to be evaluated subsequently by the older R\&K criteria. It would of course be preferable to perform larger prospective studies with the new AASM criteria. Nevertheless, against the background of the increasing importance of H-PSG and the very small number of studies investigating the FNE and RFNE in an outpatient setting, this retrospective analysis is clinically important, and the use of the R\&K criteria is not a major methodological problem. Another methodological limitation consists of the fact that only MS patients have been investigated, and the results cannot be generalized on the general population.

\section{Conclusion}

In patients suffering from SRBD and PLMD/RLS, there was a clear FNE, in PLMD/RLS albeit to a lesser extent compared to the FNE observed in the sleep laboratory. Therefore, in studies using H-PSG in an outpatient setting, two consecutive H-PSGs should be performed for a precise diagnosis. As, on the other hand, the FNE seems not sufficiently pronounced to misdiagnose SRBD or RLS/PLMD, in the clinical routine it might be considered to perform only one H-PSG to discover SRBD or RLS/PLMD. In PLMD/RLS patients, age-related changes were found in various parameters - as well in younger and in older MS patients.

In MS patients suffering from insomnia, no RFNE was found - probably due to the absence of the observation of the patient. In insomnia patients, one H-PSG seems to be sufficient for a correct diagnosis. MS patients without comorbid sleep disorders did not show an FNE (apart from an increased number of AWAs in the first night).

\section{Acknowledgments}

This is a retrospective analysis of a cross-sectional study ${ }^{20}$ published in Multiple Sclerosis Journal in 2011, which was supported by the German Research Foundation (DFG Exc 257 to FP). For the original work, Weinmann Medical Technology (Hamburg, Germany) provided two of the three PSG systems (SOMNOcheck $2 \mathrm{R} \& \mathrm{~K}$ ) used in the study. We acknowledge support from the German Research Foundation (DFG) and the Open Access Publication Fund of Charité Universitätsmedizin Berlin.

\section{Disclosure}

The authors report no conflicts of interest in this work.

\section{References}

1. Usmani ZA, Chai-Coetzer CL, Antic NA, McEvoy RD. Obstructive sleep apnoea in adults. Postgrad Med J. 2013;89(1049):148-156.

2. Woods CE, Usher KJ, Jersmann H, Maguire GP. Sleep disordered breathing and polysomnography in Australia: trends in provision from 2005 to 2012 and the impact of home-based diagnosis. J Clin Sleep Med. 2014;10(7):767-72.

3. Collop NA, Anderson WM, Boehlecke B, et al. Clinical guidelines for the use of unattended portable monitors in the diagnosis of obstructive sleep apnea in adult patients. Portable Monitoring Task Force of the American Academy of Sleep Medicine. J Clin Sleep Med. 2007;3(7): 737-747.

4. Nerfeldt P, Aoki F, Friberg D. Polygraphy vs. polysomnography: missing osas in symptomatic snorers--a reminder for clinicians. Sleep Breath. 2014;18(2):297-303. 
5. Quante M, Kaplan ER, Cailler M, et al. Actigraphy-based sleep estimation in adolescents and adults: a comparison with polysomnography using two scoring algorithms. Nat Sci Sleep. 2018;10:13-20.

6. Skarpsno ES, Mork PJ, Nilsen TIL, Holtermann A. Sleep positions and nocturnal body movements based on free-living accelerometer recordings: association with demographics, lifestyle, and insomnia symptoms. Nat Sci Sleep. 2017;9:267-275.

7. Chow CM, Wong SN, Shin M, et al. Defining the rest interval associated with the main sleep period in actigraph scoring. Nat Sci Sleep. 2016;8:321-328.

8. Wang Y, Loparo KA, Kelly MR, Kaplan RF. Evaluation of an automated single-channel sleep staging algorithm. Nat Sci Sleep. 2015;7: 101-111.

9. Auger RR, Varghese R, Silber MH, Slocumb NL. Total sleep time obtained from actigraphy versus sleep logs in an academic sleep center and impact on further sleep testing. Nat Sci Sleep. 2013;5:125-131.

10. Flemons WW, Douglas NJ, Kuna ST, Rodenstein DO, Wheatley J. Access to diagnosis and treatment of patients with suspected sleep apnea. Am J Respir Crit Care Med. 2004;169(6):668-672.

11. Bruyneel M, Ninane V. Unattended home-based polysomnography for sleep disordered breathing: current concepts and perspectives. Sleep Med Rev. 2014;18(4):341-347.

12. Le Bon O, Minner P, Van Moorsel C, et al. First-night effect in the chronic fatigue syndrome. Psychiatry Res. 2003;120(2):191-199.

13. Virtanen I, Kalleinen N, Urrila AS, Polo-Kantola P. First-Night Effect on Sleep in Different Female Reproductive States. Behav Sleep Med. 2018;16(5):437-447.

14. Le Bon O, Staner L, Hoffmann G, et al. The first-night effect may last more than one night. J Psychiatr Res. 2001;35(3):165-172.

15. Coates TJ, George JM, Killen JD, Marchini E, Hamilton S, Thorensen CE. First night effects in good sleepers and sleep-maintenance insomniacs when recorded at home. Sleep. 1981;4(3):293-298.

16. Edinger JD, Fins AI, Sullivan RJ Jr, et al. Sleep in the laboratory and sleep at home: comparisons of older insomniacs and normal sleepers. Sleep. 1997;20(12):1119-1126.

17. Sharpley AL, Solomon RA, Cowen PJ. Evaluation of first night effect using ambulatory monitoring and automatic sleep stage analysis. Sleep. 1988;11(3):273-276.
18. Wauquier A, van Sweden B, Kerkhof GA, Kamphuisen HA. Ambulatory first night sleep effect recording in the elderly. Behav Brain Res. 1991;42(1):7-11.

19. Blackwell T, Paudel M, Redline S, Ancoli-Israel S, Stone KL; Osteoporotic Fractures in Men (MrOS) Study Group. A novel approach using actigraphy to quantify the level of disruption of sleep by in-home polysomnography: the MrOS Sleep Study: Sleep disruption by polysomnography. Sleep Med. 2017;32:97-104.

20. Veauthier C, Radbruch H, Gaede G, et al. Fatigue in multiple sclerosis is closely related to sleep disorders: a polysomnographic cross-sectional study. Mult Scler. 2011;17(5):613-622.

21. McCall C, McCall WV. Objective vs. subjective measurements of sleep in depressed insomniacs: first night effect or reverse first night effect? J Clin Sleep Med. 2012;8(1):59-65.

22. Veauthier C, Paul F. Fatigue in multiple sclerosis: which patient should be referred to a sleep specialist? Mult Scler. 2012;18(2):248-249.

23. Coleman RM. Periodic movement in sleep (nocturnal myoclonus) and restless legs syndrome. In: Guilleminault C, editor. Sleeping and Waking Disorders: Indications and Techniques. New York: Addison-Wesley; 1982:265-295.

24. Sleep-related breathing disorders in adults: recommendations for syndrome definition and measurement techniques in clinical research. The Report of an American Academy of Sleep Medicine Task Force. Sleep. 1999;22(5):667-689.

25. Rechtschaffen A, Kales A, 20894: U. S. National Institute of Neurological Diseases and Blindness, Neurological Information Network. A Manual of Standardized Terminology, Techniques and Scoring System for Sleep Stages of Human Subjects. Allan Rechtschaffen and Anthony Kales. Bethesda, MD; 1968. Available from: https://locatorplus.gov/cgi-bin/Pwebrecon.cgi?DB=local\&v1=1\&ti=1,1\&Search_ $\mathrm{Arg}=0173471 \&$ Search_Code $=0359 \& \mathrm{CNT}=20 \& \mathrm{SID}=1$

26. Newell J, Mairesse O, Verbanck P, Neu D. Is a one-night stay in the lab really enough to conclude? First-night effect and night-to-night variability in polysomnographic recordings among different clinical population samples. Psychiatry Res. 2012;200(2-3):795-801.

27. Schellaert V, Labauge P, Lebrun C, et al. Psychological processes associated with insomnia in patients with multiple sclerosis. Sleep. $2018 ; 41(3)$
Nature and Science of Sleep

\section{Publish your work in this journal}

Nature and Science of Sleep is an international, peer-reviewed, open access journal covering all aspects of sleep science and sleep medicine, including the neurophysiology and functions of sleep, the genetics of sleep, sleep and society, biological rhythms, dreaming, sleep disorders and therapy, and strategies to optimize healthy sleep. The manuscript

\section{Dovepress}

management system is completely online and includes a very quick and fair peer-review system, which is all easy to use. Visit http://www. dovepress.com/testimonials.php to read real quotes from published authors. 